\title{
PASTA PHYSICS
}

The indebtedness of condensed-matter physics to pasta has previously been limited to the puzzle of fractured spaghetti: why, when the dry rods are bent to breaking point, do they break into three fragments? Richard Feynman contemplated this question, allegedly ending up with a kitchen full of broken spaghetti but no real theory ${ }^{1}$. Nickalls and Nickalls claimed to answer it in 1995 using tensor analysis ${ }^{2}$, but the full complexity was cracked, so to speak, only in a study ${ }^{3}$ published in 2005 and later awarded an Ig Nobel prize.

But pasta has yet more potential to display challenging behaviour, as Xiaodong Jia and colleagues reveal in experiments on particle segregation ${ }^{4}$. They have looked at how grains of different size and shape, including dry pasta tubes and rice, separate into distinct layers when shaken.

This phenomenon is well studied. In the 1960s, engineer J. C. Williams proposed that the rise of a large particle through a powder of finer particles during vertical vibration was a ratcheting effect caused by progressive filling of the void beneath the large particle by smaller grains during each shake ${ }^{5}$. Since then, thorough investigation of this 'Brazil-nut effect' (alluding to its operation in muesli) has resulted not in consensus but rather in a multiplication of both the observed modes of behaviour and the explanatory theories. One idea invokes convection-like movements whereby particles rise in the centre of the container and sink at the walls ${ }^{6}$. Under some circumstances, the large grains can sink rather than rise: the reverse Brazil-nut effect ${ }^{7,8}$.

Jia and colleagues claim to bring something new to this field by focusing on the geometric rather than the dynamical aspects of the problem. That is to say, they disregard aspects such as the energetics of collision and friction between particles, and look only at the structures that result from the effects of particle size and shape on their mobility.

They use a simple simulation algorithm in which particles follow random walks, as if fluidized, subject to the constraints of a bias against upward motion (to simulate settling under gravity) and prohibition of particle overlap. Where the particles end up depends on how easily, given their geometries and those of their neighbours, they can shift around. Considering mixed beds of coarse grit, fine sand, rice and pasta tubes, the researchers find they can reproduce the trends in segregation observed in their experiments on the same materials shaken within a pseudo-twodimensional vertical cell. In particular, the rate of segregation depends on how readily the smaller particles can infiltrate the voids between bigger particles.

Pasta here, like rice, offers simply a convenient medium for experiment:

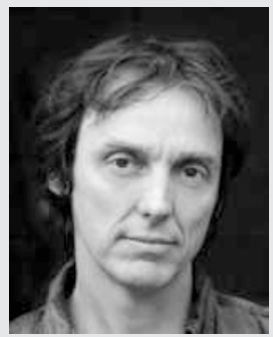

PHILIP BALL

reasonably monodisperse in size and shape, and cheap. But there's every reason to take the use of such foodstuffs seriously, because food science and packaging is one of the most pertinent applications for the science of granular systems. Indeed, it is reasonable to consider these experiments precursors to an exploration of that familiar mixture of ovoids, wrinkled ovoids, circular flakes and fine particulates better known as muesli.

References

1. Sykes, C. (ed.) No Ordinary Genius: The Illustrated Richard Feynman 181 (W. W. Norton, 1994).

2. Nickalls, O. \& Nickalls, R. New Scientist 145, 52 (1995)

3. Audoly, B. \& Neukirch, S. Phys. Rev. Lett. 95,095505 (2005).

4. Caulkin, R., Jia, X., Fairweather, M. \& Williams, R. A. Phys. Rev. E 81, 051302 (2010).

5. Williams, J. C. \& Shields, G. Powder Technol. 1, 134-142 (1967).

6. Knight, J. B., Jaeger, H. M. \& Nagel, S. R. Phys. Rev. Lett. 70, 3728-3731 (1993).

7. Shinbrot, T. \& Muzzio, F. J. Phys. Rev. Lett. 81, 4365-4368 (1998).

8. Hong, D. C., Quinn, P. V. \& Luding, S. Phys. Rev. Lett. 86, 3423-3426 (2001).

\section{Surface attraction}

\section{A new route to layer-by-layer assembly of metal-organic framework thin films affords highly ordered and controllable surfaces with potential in chemical sensing and catalyst applications.}

\section{Mark A. Green}

$\mathrm{M}$ etal-organic frameworks (MOFs) are an emerging class of porous materials; with low densities and high specific surface areas they offer new opportunities in applications such as absorption, separation, sensing and shape-selective catalysis ${ }^{1}$. The fabrication of nanoscale MOF films is a particularly valuable objective towards their commercial utilization. Writing in Nature Materials, Makiura et al. ${ }^{2}$ report the implementation of a metalloporphyrin as a building unit that affords highly ordered and controllable layers of MOFs using a modified
Langmuir-Blodgett procedure. The apparent ease with which these complex, highly ordered nanofilms can be constructed at ambient temperatures means it is a method that promises to facilitate a new generation of robust and structurally integral ultrathin films. 